\title{
Based on Delaunay Triangulation DEM of Terrain Model
}

\author{
Yan Li \& Lianhe Yang \\ College of Computer application technology, Tianjin Polytechnic University \\ Tianjin 300160, China
}

Tel: 86-22-24552438Ｅ-mail:boasara123@163.com

\begin{abstract}
In this paper, DEM data acquisition methods were introduced, and the current use of the most popular data srtm dem 3D terrain modeling, algorithm-based Delaunay, through the establishment of triangular mesh network data model, so as to achieve modeling results.
\end{abstract}

Keywords: Delaunay, Terrain Model, Triangulation, DEM

\section{Introduction}

Digital Terrain Model (Digital Terrain Model acronym DTM) is arbitrary use of a large number of coordinates in three-dimensional $\mathrm{x}, \mathrm{y}, \mathrm{z}$ coordinates of the point on the ground for a form of Statistics said that the terrain surface morphology is the number of attribute information is a space location characteristics and attributes of the terrain described the figures, initially for the automatic design of highway proposed. With the world of computer technology and the rapid development of 3-D visualization technology into the traditional static two-dimensional map of the three-dimensional terrain modeling makes a Geographic Information System (GIS) and digital mapping, a new field of study. DTM is the basis of geographic information system data, mainly used to describe the ground state of ups and downs, the terrain can be used to extract various parameters such as slope, aspect, roughness, and Visibility analysis, watershed generation applications such as structural analysis. Therefore, the DTM in land use analysis, and rational planning, forecasting flood danger, as well as military navigation and missile guidance systems, and combat electronic sand table, and other fields are widely used. Digital Terrain Elevation Model mainly contains the attributes of surface morphology, as well as other attributes, such as slope, aspect and so on. Terrain Elevation attribute is the basis of the model attributes, other elements of the terrain elevation attributes can be directly or indirectly receive, digital elevation model (Digital Elevation Model, acronym DEM) acts as a digital terrain on the main study. DEM is that the number of regional terrain, elevation $\mathrm{Z}$ coordinates on the plane $\mathrm{X}$, $\mathrm{Y}$, the two variables of continuous function of a limited discrete said, a series of ground from the X, Y location and elevation linked by some Organization structure with the actual terrain features that the spatial distribution model, it is a spatial information system an important component part.

\section{Acquisition DEM data}

DEM access to commonly used data there are mainly two ways, one is through the aerospace remote sensing image of the three-dimensional, as measured by this method has been updated topographic mapping and the most effective means to obtain the images are high-precision a wide range of data, but the relative and absolute precision accuracy are far too low.

Can also interfere radar and laser scanners, and other sensor data to obtain high-precision, high-resolution DEM, but generally have a high cost. Another method is to use the existing topography scan digital contour lines, access to elevation data generated DEM, as data generated high efficiency, low cost, topographic maps related experiment, measuring use of this method, and can achieve the desired results. This paper is based on the popular $90 \mathrm{~m}$ resolution srtm dem data.

According to the original data set collection of the different ways, digital elevation modeling There are four main ways: modeling methods based on points, based on the triangle modeling approach, based on the grid and triangle modeling methods used in conjunction with the grid Hybrid modeling method. Based on the point of data modeling method is through the establishment of a point on every level plane to the point that in the vicinity of a small region, but between each plane is not continuous, it is not true method of modeling can be used. Grid-based modeling approach is the point set of equidistant square surface modeling. The square, rectangular shape, as well as other arbitrary polygon can be decomposed into a number of different shapes of triangles, based on the triangle modeling applies to all data structure. The triangular in shape and size on the high degree of flexibility, all triangles modeling method can be applied to a variety of terrain, this approach is gaining more and more attention, and the surface topography is the main method of 
modeling. In this paper, using the rules for the grid DEM model, so it is necessary to study the rules is how to mesh model has become a triangular model, terrain modeling. Rules grid model is usually square; it can also be a rectangular grid, and other rules. Grid rules will be divided into regional space cut rules grid units, each grid cell of a numerical counterpart. Mathematics can be expressed as a matrix; in the computer realization is a two-dimensional array. Each grid cell or array element, the corresponding value of a height, as shown in Table1.

For each of the numerical grid there are two explanations. One is that the values in the cells for topographical maps for each data point elevation value; another kind of cell in the view that the values for each grid point of elevation center coordinates of the grid unit, or the average height of This would require the use interpolation method was used to calculate coordinates for each point of elevation, the need for grid computing is not the center of the elevation of data points. Rules of the elevation grid matrix, can be easily processed by computer to calculate contour, slope, aspect, slope shadow and automatic extraction basin topography, making it the most widely used DEM format, in many countries the DEM Data are rules grid data matrix form. Grid DEM shortcoming is that it can not accurately that the structure and details of terrain, in order to avoid these problems, the data can be added topographical features, such as terrain features, ridgelines, and the bottom line, fault lines, with a description of topographic structure. Grid DEM Another disadvantage is that the volume of data to the data management brought inconvenient, under normal circumstances should be compressed storage. DEM data compression lossy compression and divided into two lossless compressions. Lossless compression can be used ordinary raster data compression methods, such as run-length coding, block codes, but because of DEM data reflects the undulating terrain of continuous change, but difficult to achieve common compression very good results, sometimes, without taking into account the details Under the circumstances, can be carried out on the grid DEM lossy compression, the wavelet transform is commonly used compression.

\section{Delaunay modeling algorithm based on topography}

\subsection{Delaunay Algorithm}

Delaunay main segmentation algorithm - merging algorithm, and point by point insertion method of triangulation algorithm, the algorithm of the current triangulation using less, segmentation - merging algorithm relatively complex, so little data in the cases, point by point insertion method is the most simple, occupying less memory approach. Point by point by the insertion method is the counterpart of the Voronoi polygon edge adjacent to the three-point triangle linking a triangular irregular network (TIN), the three corresponding points Voronoi adjacent polygons are the culmination of a public, and this is also vertex Delaunay Triangulation circumcircle the center. Voronoi polygon by polygon composed of a group of continuous, polygon boundary by linking the two neighboring segments of the perpendicular bisector line. Delaunay Triangulation is shared with an adjacent Voronoi polygon edges relevant point connection from the triangle, as shown in Figure 1

Delaunay point by point interpolation algorithm for the basic rules of each triangle is not included within the circumcircle other coordinates; Figure 2 shows the different circumstances in the new coordinate's interpolation point output. While this is not the best method of triangulation, but on the whole tended to the best, for the most suitable terrain modeling choice.

\subsection{Triangle Network Modeling}

Adopt rules TIN grid data generation, there are two general ways. One is the direct use of a grid square or two diagonal connections, broken down into a series of rules triangle. But this approach generated by the triangular network, is a very casual, shown in Figure3. A group of the same data grid will generate several different types of triangulation to join the elevation of the coordinate point after that the outcome will be a big difference.

Another method is for all the coordinate point screening, select important points (VIPs), and through the Delaunay triangulation method to a triangular irregular network. One approach is calculated by comparing the importance of grid points to retain important grid network. The important point is 4 adjacent to determine the grid, according to the adjacent eight-point elevation of the value of the decision of whether or not the focal point for point. Coordinate point is the importance of value through its elevation with eight points adjacent to compare elevation, when the difference exceeds a certain threshold value of the coordinates little retained, be retained as a triangulation point vertex generation Delaunay Triangulation. Figure 4 shows, to the point P ICC straight AE, CG, BF, DH space distance, then calculate the average of four distances. If the average exceeds the threshold value, P points to an important point, the retention or removal of the $P$ point.

Select important points can be inserted through the iterative method greed important point on the line optimization. The basic steps are as follows:

(1) DEM on the coordinates of points on the border all constructed through Delaunay Triangulation.

(2) Calculated contained within each triangular grid coordinates from the point of the triangle of the greatest distance, as the triangle of "candidate point." 
(3) To compare all Triangle "candidate points," Height values, the greatest value will elevation inserted triangulation points, a new triangulation, calculation and all the new triangulation triangle "candidate point."

(4) Repeat the process (2)(3), until the termination conditions.

DEM has been carried out through the experiment; substituting greed insertion method avoids the search for a "critical points" in the process of double counting, increase the speed of the algorithm, and good retention characteristics of the terrain. Obviously, the choice of methods and VIPs compared to the direct method, though more complex, but the terrain data redundancy can be avoided in large-scale visualization of the details of terrain modeling, VIPs method is widely used.

\section{Conclusion}

Above methods, and use $\mathrm{VC}++6.0$ on the computer realization of the terrain modeling experiments show that this method is effective, in fact only terrain visualization of the basic work, the quality of the data will directly affect the result will be displayed. The volume of data smaller topographic maps, generated Delaunay Triangulation, high efficiency, and will get very good results. Can be predicted with computer hardware technology, such as the development of computer graphics, as well as in all walks of life in the application of the in-depth, three-dimensional terrain visualization of the direction of development, will be displayed towards the "virtual reality" of the direction of development of functional performance and geographic information systems will have a close relationship, and to deal with dynamic updates. 3D terrain visualization technology applications prospects are very bright.

\section{References}

Cheng Penggen, Liu Shaohua, Wang Beechen Honghua. (2004). Construction of three-dimensional geological model of the Research and Application. Journal of Jilin University: Earth Sciences. 2004. 4.

Jill McCoy, Kevin Johnston, e.i. (1997). Using ArcGIS Spatial Analyst. Publisher: IS By ESRI.

Schroeder W J.Avila L S, Hoffman W. (2000). Visualization with VTK: A Tutorial. IEEE Computer Graphics and Applications.

Wang Ruchuan, Zou Beiji. (2002). Computer Graphics. Posts And Telecommunications News Publishing House.2002. 7 .

Xu Qingrong, Du Daosheng, Huang Wei. (1997). Computer Mapping Principle. Mapping Wuhan University of Science and Technology Publishing House.

Table1. The corresponding value of a height

\begin{tabular}{|l|l|l|l|l|l|l|l|l|l|}
\hline 619 & 634 & 639 & 638 & 638 & 638 & 641 & 641 & 642 & 643 \\
\hline 646 & 644 & 645 & 648 & 649 & 650 & 652 & 654 & 655 & 655 \\
\hline 661 & 664 & 666 & 667 & 666 & 667 & 666 & 667 & 667 & 670 \\
\hline 673 & 675 & 675 & 675 & 673 & 673 & 675 & 679 & 678 & 677 \\
\hline 682 & 684 & 686 & 686 & 687 & 685 & 687 & 687 & 689 & 690 \\
\hline 692 & 693 & 694 & 694 & 693 & 695 & 695 & 691 & 694 & 693 \\
\hline 692 & 700 & 708 & 691 & 661 & 669 & 681 & 662 & 675 & 697 \\
\hline 718 & 722 & 715 & 717 & 718 & 703 & 703 & 717 & 725 & 711 \\
\hline 645 & 652 & 644 & 636 & 649 & 663 & 679 & 701 & 715 & 708 \\
\hline
\end{tabular}

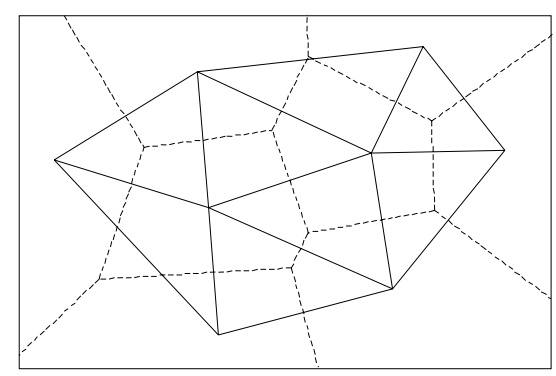

Figure 1. Voronoi polygon 


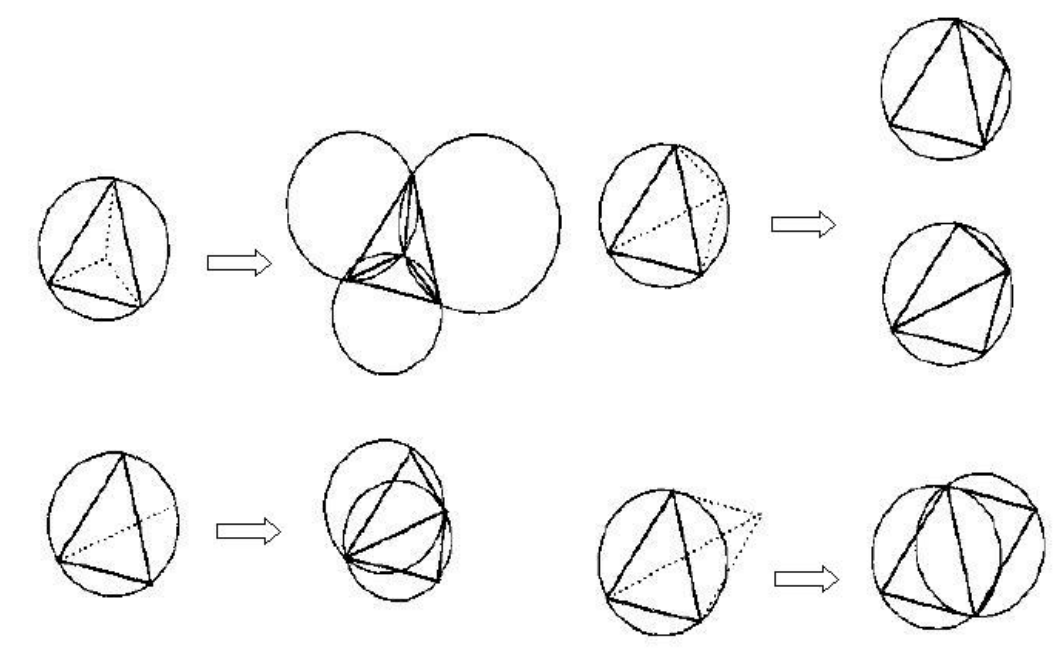

Figure 2. Interpolation of the new coordinates of the points

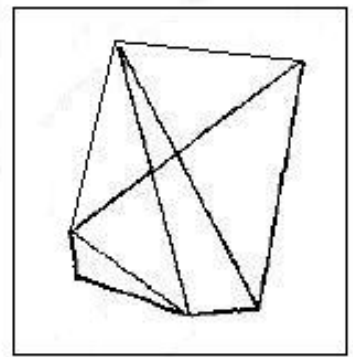

(a)

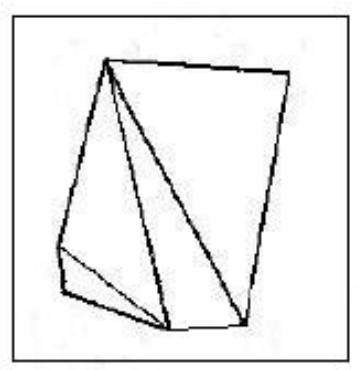

(b)

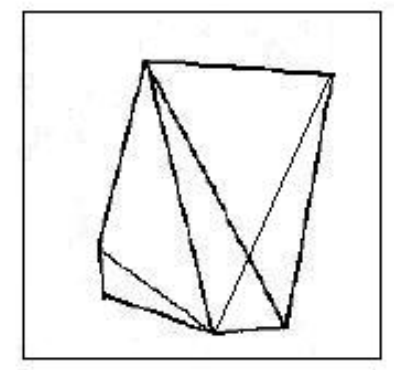

(c)

Figure 3. Rules triangle

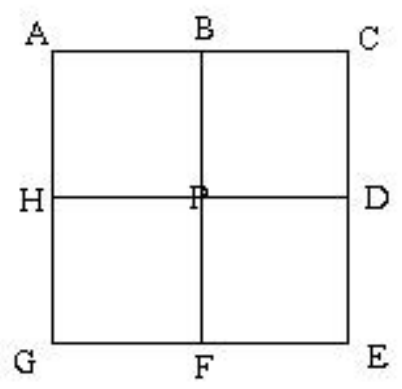

Figure 4. Eight neighborhoods 